\title{
The bifunctional dihydrofolate reductase thymidylate synthase of Tetrahymena thermophila provides a tool for molecular and biotechnology applications
}

\author{
Lutz Herrmann ${ }^{1}$, Ulrike Bockau ${ }^{1,2}$, Arno Tiedtke ${ }^{2}$, Marcus WW Hartmann*1 \\ and Thomas Weide ${ }^{1}$
}

Address: ${ }^{1}$ Cilian AG, Johann-Krane Weg 42, D-48149 Münster, Germany and ${ }^{2}$ Institut für allgemeine Zoologie und Genetik, Universität Münster, Schloßplatz 5, D-48149 Münster, Germany

Email: Lutz Herrmann - herrmann@cilian.de; Ulrike Bockau - bockau@cilian.de; Arno Tiedtke - tiedtke@uni-muenster.de; Marcus WW Hartmann* - hartmann@cilian.de; Thomas Weide - weide@cilian.de

* Corresponding author

Published: 20 March 2006

BMC Biotechnology2006, 6:2I doi:10.1186/1472-6750-6-2I

This article is available from: http://www.biomedcentral.com/I472-6750/6/2I

(c) 2006Herrmann et al; licensee BioMed Central Ltd.

This is an Open Access article distributed under the terms of the Creative Commons Attribution License (http://creativecommons.org/licenses/by/2.0), which permits unrestricted use, distribution, and reproduction in any medium, provided the original work is properly cited.
Received: 05 October 2005

Accepted: 20 March 2006

\begin{abstract}
Background: Dihydrofolate reductase (DHFR) and thymidylate synthase (TS) are crucial enzymes in DNA synthesis. In alveolata both enzymes are expressed as one bifunctional enzyme.

Results: Loss of this essential enzyme activities after successful allelic assortment of knock out alleles yields an auxotrophic marker in ciliates. Here the cloning, characterisation and functional analysis of Tetrahymena thermophila's DHFR-TS is presented. A first aspect of the presented work relates to destruction of DHFR-TS enzyme function in an alveolate thereby causing an auxotrophy for thymidine. A second aspect is to knock in an expression cassette encoding for a foreign gene with subsequent expression of the target protein.
\end{abstract}

Conclusion: This system avoids the use of antibiotics or other drugs and therefore is of high interest for biotechnological applications.

\section{Background}

Tetrahymena thermophila is a ciliated eukaryotic unicellular organism belonging to the regnum of protozoa and bearing two nuclei, a transcriptionally silent, diploid germline micronucleus (MIC) and a transcriptionally active, polyploid somatic macronucleus (MAC)[1]. In 1923, when Nobel Laureate Andre Lwoff succeeded in growing Tetrahymena in pure culture, the basis for exploiting this alveolate as a model organism was laid. Milestone discoveries made in $T$. thermophila are the discovery of dynein motors[2], telomeres[3], RNA-mediated catalysis[4], telomerase[5] and the function of histone acetyltransferases in transcription regulation[6]. Within the last decades molecular biological techniques have been developed to alter T. thermophila's genome and proteome: DNA transfection methods range from microinjection[7] and electroporation [8] into the MAC to biolistic bombardment of MIC and MAC[9]. Episomal plasmids based on an rDNAreplicon are available[10], as well as knock out/-in techniques based on homologous recombination $[11,12]$.

On protein level heterologous expression of related species has been performed $[13,14]$ and also endogenous proteins were silenced by a novel antisense-ribosometechnique[15]. The biotechnological potential of T. thermophila has been proven in numerous publications, dem- 
A

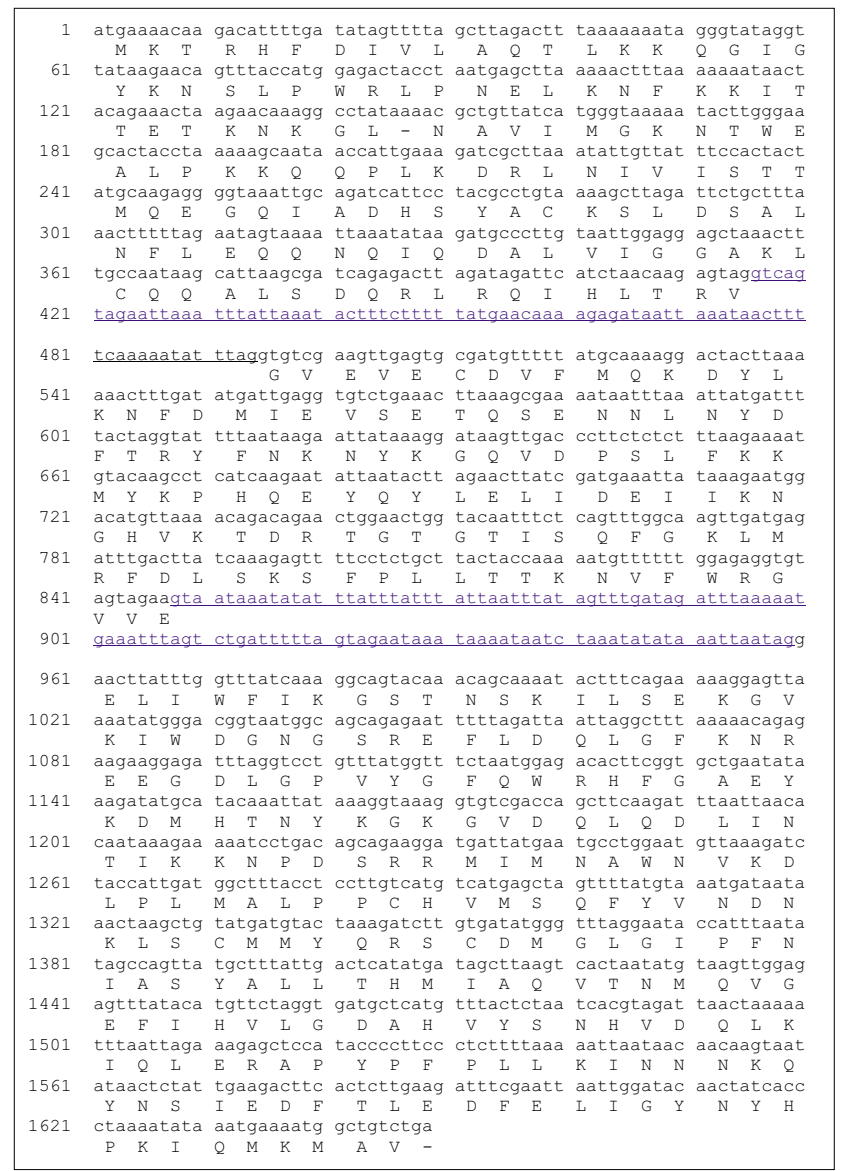

B

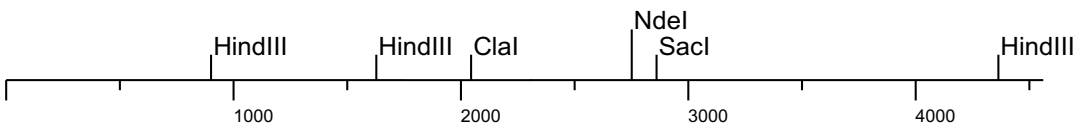

DHFR-TS gene (4556 bp)

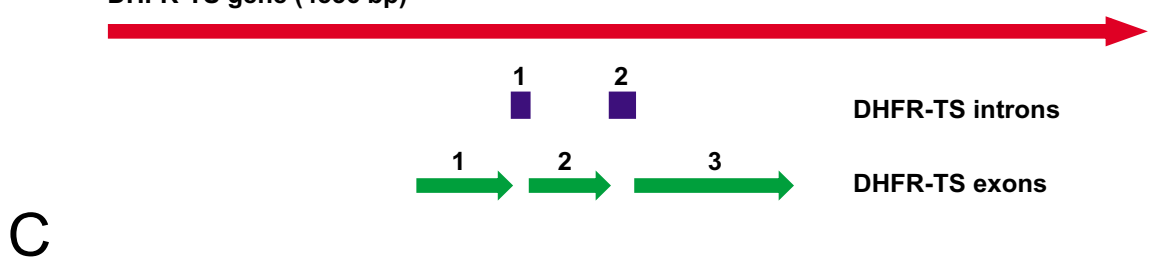

PCR fragments / primers

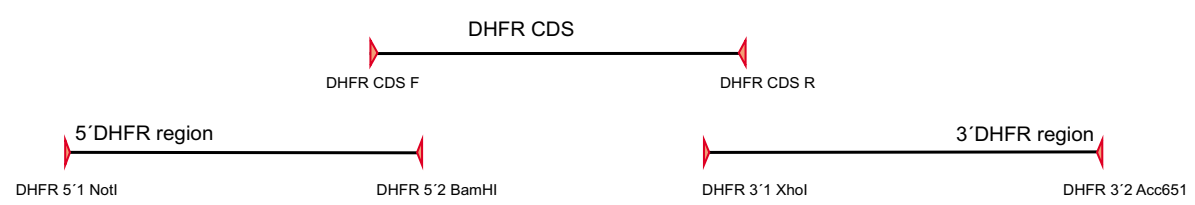

Figure I

Genomic structure of T. thermophila bifunctional DHFR-TS enzyme. A: The DNA sequences of the gene and the cDNA as well as the deduced amino acid sequence are shown on the left (introns underlined, blue). B: The DHFR-TS gene structure of $T$. thermophila consists of three exons (green) and two introns (blue). C:Overview of primer pairs to amplify DNA for homologous integration and to amplify the CDS. 


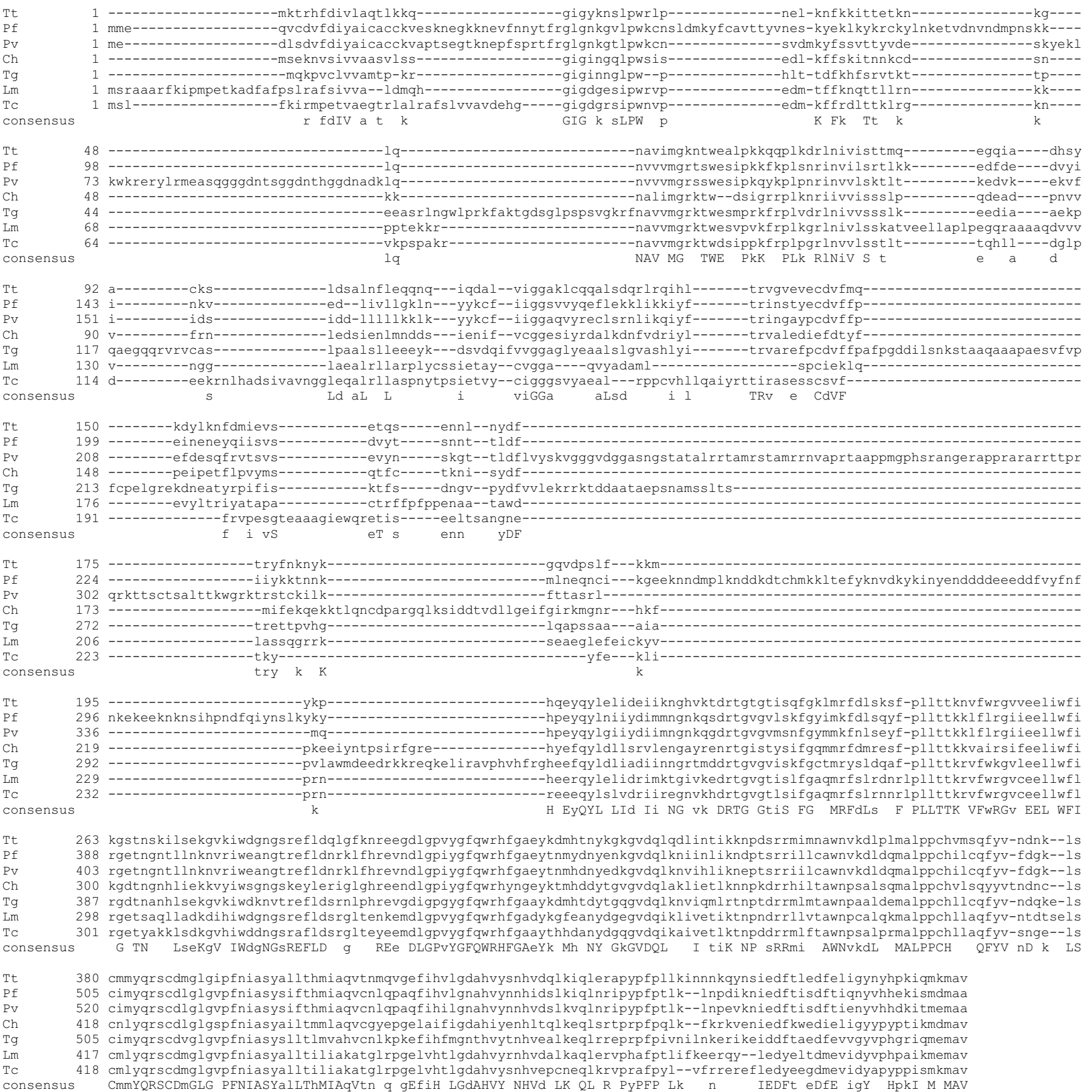

\section{Figure 2}

Comparison of the T. thermophila DHFR-TS bifunctional enzyme to other protozoa. Amino acid sequence comparison of Tetrahymena thermophila (Tt), Plasmodium falciparum (Pf), Plasmodium vivax (Pv), Cryptosporidium hominis (Ch), Toxoplasma gondii (Tg), Leishmania major (Lm) and Trypanosoma cruzi (Tc) DHFR-TS bifunctional enzyme.

onstrating fast growth, high biomass, fermentation in ordinary bacterial/yeast equipment, up-scalability, existence of cheap and chemical defined media [16-18].

Although known and analysed for decades, only a few markers have been described for T. thermophila. So far there are ribosomal point mutation mediated resistances, a plasmid based neomycin resistance [19] and a beta-tubulin selection marker making use of an inducible promoter in combination with mutated tubulins being resistant or sensitive to the mitotic drug taxol[20]. Yet no true auxo- 
trophic marker is available that permits selection without the use of antibiotics or drugs.

Critical enzymes in pyrimidine biosynthesis are dihydrofolate reductase (DHFR) and thymidylate synthase (TS). DHFR catalyses the production of tetrahydrofolate from dihydrofolate; TS is in charge of transferring a methylgroup from N5, N10-methylene-tetrahydrofolate to dUMP thereby generating dTMP and tetrahydrofolate. These enzymes being crucial for pyrimidine synthesis have been used as auxotrophic markers in various systems by targeted gene disruption but also a number of inhibitors (antifolates) have been developed as anti-cancer drugs[21]. In animals, fungi and eubacteria the DHFR and TS gene are separately translated, whereas plants, alveolata and euglenozoa have a bifunctional fusion gene with both enzyme activities combined in one protein ("DHFRTS").

The occurrence of the bifunctional enzyme in T. pyriformis has been postulated in 1984 and 1985 but no functional or even molecular biological analysis had been performed. A partial amino acid sequence of DHFR-TS of a non determined "T. pyriformis-like strain" has been published in 2002[22], but this work is lacking any proof of linkage to the described partial cDNA to enzyme function. Here we present a first characterization of the T. thermophila DHFR-TS gene including gene structure and functional data on the enzyme and data on in vivo function. For the first time we show that the T. thermophila DHFRTS locus provides an auxotrophic marker system that enables monitoring of allelic assortment processes. As PCR based approaches always have to cope with wildtype alleles present in the MIC, the DHFR-TS auxotrophy system is able to deliver direct proof of the allelic assortment to be completed: Only in the case of all wildtype alleles in the MAC having been substituted by the knock out construct the auxotrophy will occur. Combining this methodology with a knock in, a new, stable and useful strain to express recombinant enzymes or proteins has been generated.

\section{Results}

Tetrahymenathermophila is a free-living ciliated protozoan that has become increasingly interesting as an excellent expression system. It is one of the best characterised unicellular eukaryotes and its genome has been sequenced in its entirety (The Institute for Genomic Research[23]). These features form the basis for using T. thermophila in future biotechnology applications. However, appropriate marker systems that are essential tools for genetic manipulations are limited. The enzymes dihydrofolate reductase (DHFR) and thymidylate reductase (TS) play a crucial for pyrimidine synthesis, therefore both enzymes have been used as auxotrophic marker-systems in various species by targeted gene disruption. The aim was to establish such a

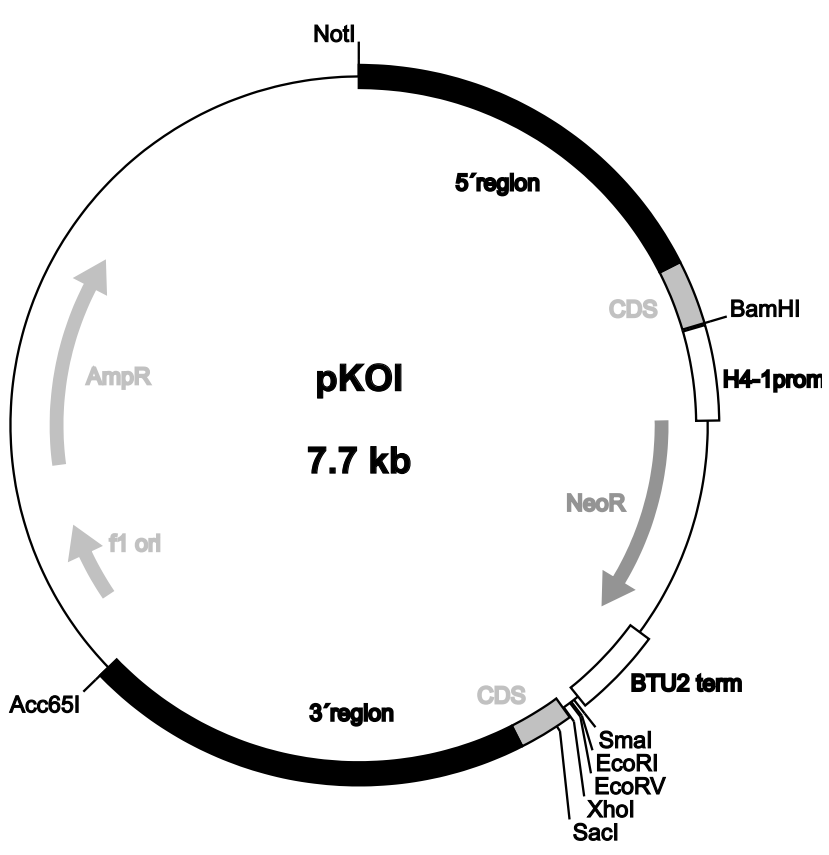

Figure 3

pKOI: The basis for a DHFR-TS knock out construct. Figure 3 shows the knock out construct used for a targeted knock out of the DHFR-TS gene in T. thermophila. It consists of 3 ' and 5' flanking regions of the T. thermophila DHFR-TS gene and parts of its coding sequence (CDS), disrupted by a functional neomycin cassette conferring resistance to paromomycin. For more details refer to material and methods section.

DHFR marker system for T. thermophila. Interestingly, the bifunctional DHFR-TS has been as taxonomic tool to set up phylogenetic trees for years. These approaches led to a very rudimental DHFR-TS amino acid fragment sequence of a non determined "T. pyriformis-like strain". By using this incomplete information and data from the entire $T$. thermophila genome it was possible to determine the whole sequence of the bifunctional DHFR-TS MAC gene in T. thermophila. The T. thermophila DHFR-TS gene and its flanking regions were amplified by using the primer pairs DHFR 5'1F NotI, DHFR 5'2R BamHI for the 5'region non coding region, the primer pair DHFR 3'1F XhoI: 5, DHFR 3'1R Acc65I for the 3 'region and the primers DHFR CDS$\mathrm{F}$, DHFR CDS-F for amplification of the coding region.

\section{The T. thermophila DHFR-TS gene structure}

The comparison of the amino acid sequences of related species to the translated coding region of the DHFR-TS structure gene revealed the corresponding cDNA (see figure 1). Alignment with the cDNA with the gene revealed the intron exon boundaries. Figure 1 summarises the results and shows the MAC gene structure with two 


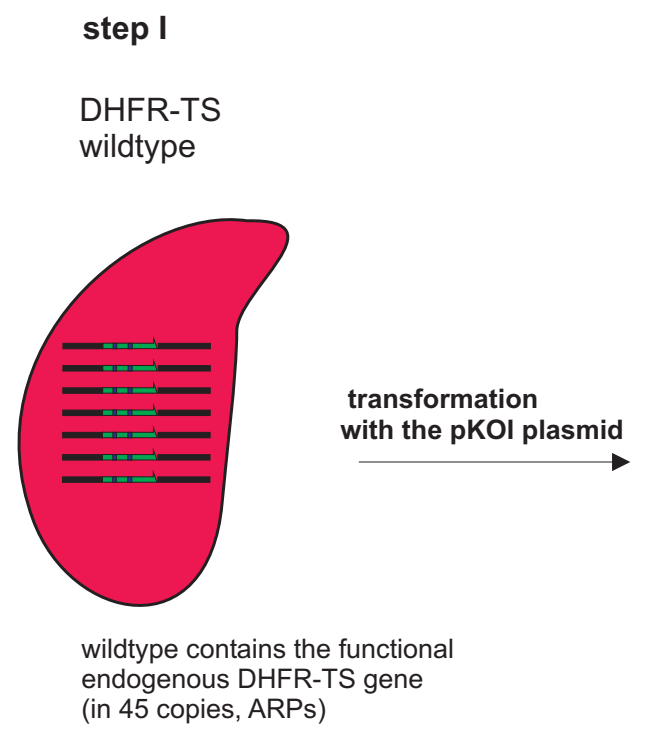

step I

step II

DHFR-TS

transformant

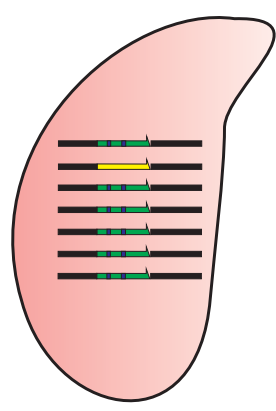

transformant,

contains at least

one recombinant

DHFR-TS k.o. allel step III

DHFR-TS

deficient strain

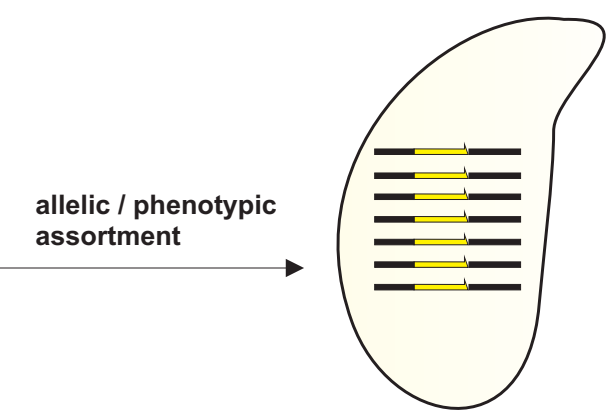

loss of DHFR-TS activity because of complete replacement of the functional DHFR-TS gene by the k.o. allel

\section{Figure 4}

Generation of DHFR-TS deficient strains by allelic assortment. Wildtype strains are transfected with pKOI. In one copy of the 45 ARPs the endogenous DHFR-TS gene is substituted by the knock out construct (step 2). By amitotic division of the MAC and increasing selection pressure clones will arise, that have sorted out all endogenous DHFR-TS genes and retain only recombinant and defect DHFR-TS genes (step 3).

introns, the corresponding DNA sequence and the amino acid sequence of the T. thermophila DHFR-TS.

\section{Sequence alignment of the T. thermophila DHFR-TS enzyme to other alveolates}

The alignment of the DHFR-TS between the alveolates Tetrahymena thermophila, Plasmodium falciparum, Plasmodium vivax, Cryptosporidium hominis and Toxoplasma gondii (Tt, $\mathrm{Pf}, \mathrm{Pv}, \mathrm{Ch}, \mathrm{Tg}$ in figure 2) illustrates a $50-60 \%$ degree of identity between the proteins. This is also the case if the ciliate DHFR-TS is compared to the analogous enzyme of Leishmania major and Trypanosoma cruzi (Lm, Tc in figure 2). This is almost due to the highly conserved thymidylate synthase part of the bifunctional enzymes. A highly conserved histidine is found at position 198, suggesting that the amino acids $198-485$ represent the thymidylate synthase part of the bifunctional enzyme. Much more heterogeneity is found in the N-terminal part of the bifunctional enzyme that consists of the DHFR part and a linker site. The alignment reveals that almost all enzymes bear one or more individual, non-homologue amino acid stretches (figure 2).

\section{Proper integration of the knock out construct into the DHFR-TS locus and auxotrophy for thymidine}

In order to verify that only one DHFR-TS activity is present in $T$. thermophila and that the sequence given in figure 1 corresponds to this activity a construct for knocking out the DHFR-TS gene was made. By inserting the flanking regions of the DHFR-TS gene into a pBS II SK backbone we created a plasmid for stable integration into the ciliate genome. The neo2 cassette of pH4T2[19] was used to monitor the successful uptake of this plasmid by selection against paromomycin. Figure 3 shows that the neo 2 cassette is flanked by the $\sim 1.5 \mathrm{~kb}$ fragments of the $5^{\prime}$ and $3^{\prime}$ parts of the non-coding regions of the DHFR-TS gene, respectively. Because the pBS backbone lacks an appropriate origin of replication, paromomycin resistant $T$. thermophila clones argue for proper homologous recombination in the DHFR-TS gene locus.

The most convincing evidence for the correct integration into the DHFR-TS locus is a loss of the DHFR-TS activity in the transformed strain. However, in the case of T. thermophila this requires the complete replacement of all chromosomal DHFR-TS wildtype alleles ( 45 ARPs) by the ARPs that includes the knock out cassette. We achieved this by allelic assortment, a phenomenon summarised in figure 4 . This allelic or phenotypic assortment is based on randomised distribution of the MAC chromosomes units (ARPs) during mitosis. In order to force the assortment process into the desired direction - namely into the recombinant resistance gene - the transformed cells were cultivated for at least 3-4 weeks using increasing concentrations of the drug paromomycin. Single clones were isolated and tested for DHFR-TS deficiency by 

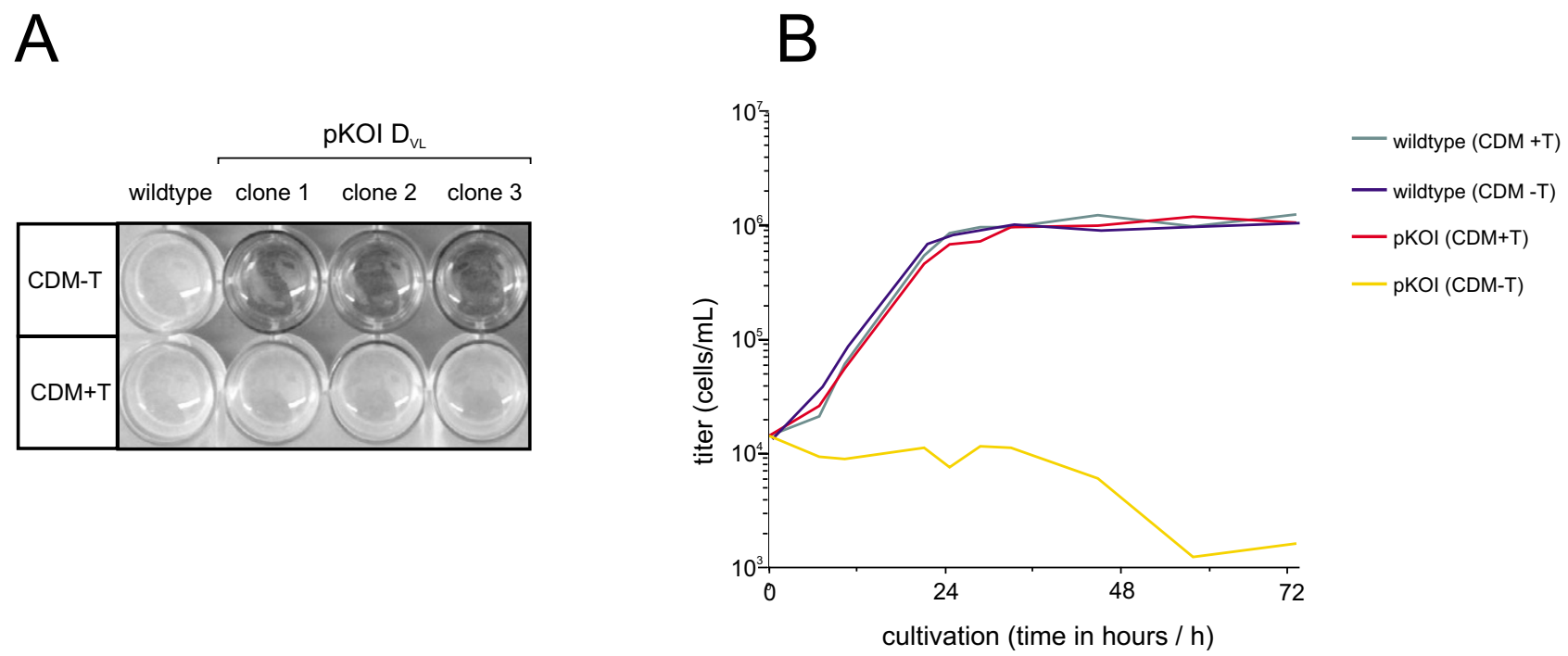

\section{Figure 5}

Growth of DHFR-TS deficient T. thermophila compared to wildtype. A: Selection of DHFR-TS knock out cells by growth on thymidine. T. thermophila cells with disrupted DHFR-TS gene (clone I-3) do not grow without the presence of thymidine (CDM-T), whereas wildtype cells do. Addition of thymidine to the medium (CDM+T) recovers growth. B: Growth kinetics of DHFR-TS knock out cells compared to wildtype cells in media with or without thymidine show, that the knock out strain $(\mathrm{pKOI})$ is growing as fast as wildtype cells on thymidine supplemented media (CDM+T). Knock out cells die without thymidine present (CDM-T). The curves are calculated by mean values of at least three independent experiments.

using a minimal chemical defined medium (CDM) with $(+\mathrm{T})$ and without thymidine $(-\mathrm{T})$. It could be shown that DHFR-TS knock out clones are real auxotrophic strains. The mutants are able to grow in CDM with thymidine like wildtype strains or strains with an incomplete allelic assortment. In CDM lacking thymidine they are unable to grow (figure 5).

\section{Functional addition of an expression cassette to the knock out construct}

The knockout of the endogenous DHFR-TS gene of T. thermophila also provides the possibility to knockin a further foreign gene that can be expressed heterologously in the DHFR-TS knock out strains. Therefore we named our constructs pKOI (pKOI: knock out and knock in). To illustrate this knock out/-in concept we constructed the pKOI $\mathrm{D}_{\mathrm{VL}}$ plasmid. It consists of a pKOI backbone with an additional expression cassette that encodes the first 115 amino acids (aa) of the precursor sequence of the PLA $_{1}$ gene and the mature human DNase I (aa 23 to 281). The PLA 1 pre/ pro-peptide (aa 1 to 110 ) has significant similarity to members of the cathepsin L family and mediates secretion into the medium[24]. The five additional amino acids (aa 111 to 115) should ensure an optimal cleavage of the pro PLA $_{1}$-DNaseI fusion protein by endogenous pro-peptidases. In contrast to the neo 2 cassette the expression of the ppPLA $_{115}$-DNase I fusion protein is regulated by the inducible MTT1 promoter[25]. The inducible system was selected because it allows a clear discrimination between the DNase activity of heterologously expressed recombinant human DNase I and the basal activity due to at least two endogenous DNases. The transformation, selection of positive clones and the directed allelic assortment were done as described in the material and methods section.

Furthermore, we tested the correct and complete integration of both expression cassettes (neo2 and the DNase I) in the DHFR-TS locus by a PCR approach (figure 6). In order to demonstrate the pKOI concept, cells of these DHFR-TS knock out strains carrying the ppPLA ${ }_{115}$-DNase expression cassette were treated with and without Cadmium. Only induced strains showed an elevated DNase activity in the supernatant (Figure 7). To confirm this enzymatic data a specific antiserum against human DNase I was used to analyse the cell extracts and the supernatant of these human DNase expressing DHFR-TS knock out strains by Western blot. The results illustrate that the DHFR-TS knock out strains are capable of expressing and secreting the functional recombinant human protein (Figure 8).

\section{Discussion}

The ciliated protozoan $T$. thermophila features two nuclei, a somatic macronucleus (MAC) and a genetic micronucleus (MIC) offering different possibilities of manipulat- 


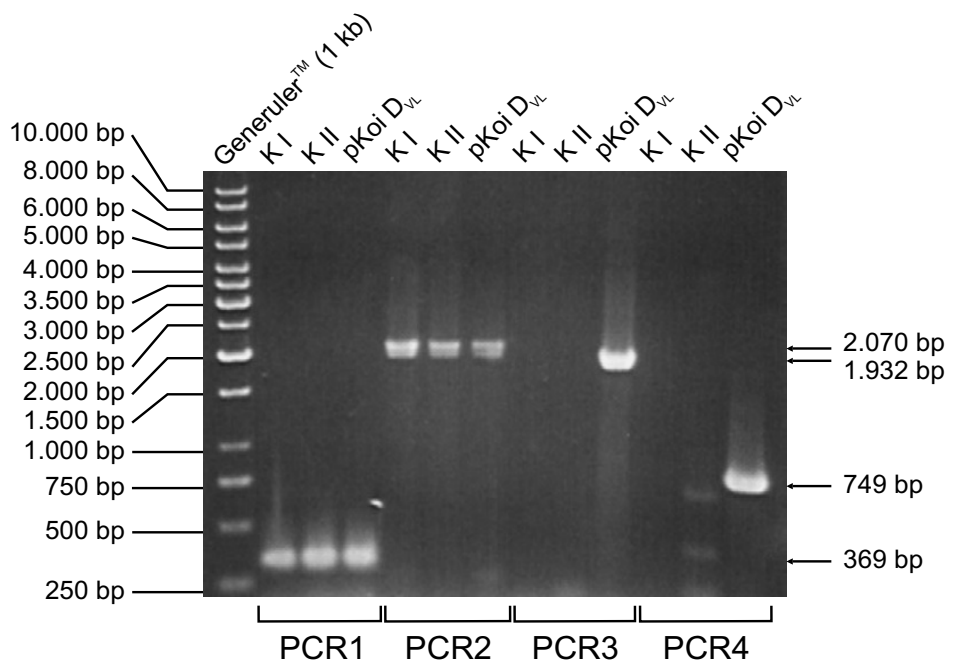

DHFR-TS gene sructure

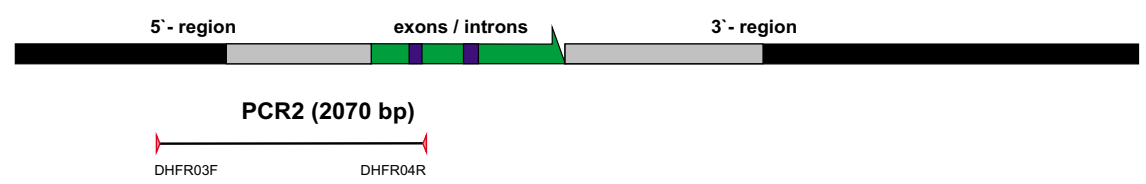

pKOI DvL plasmid

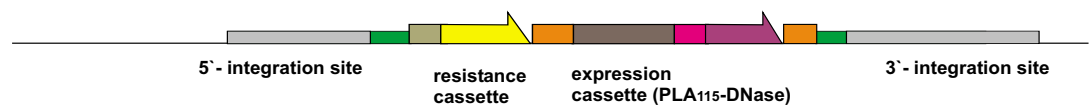

DHFR-TS knock out by knock in (integration)
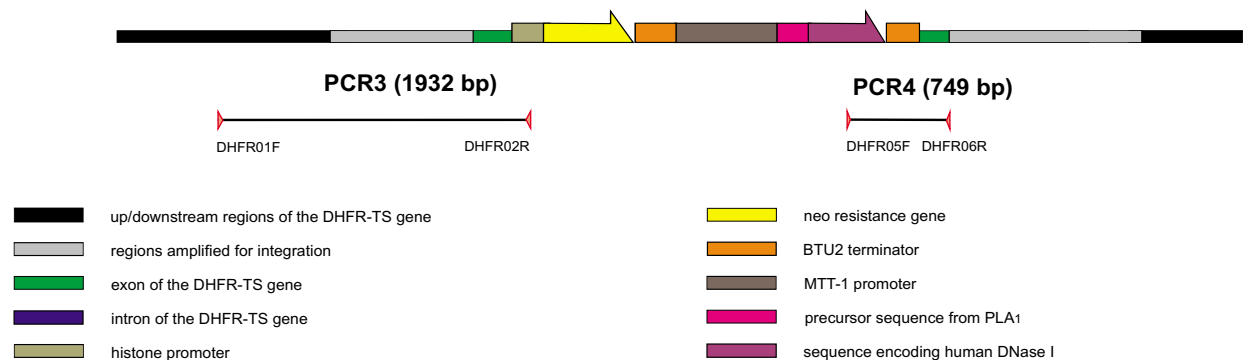

Figure 6

Proper integration of DHFR-TS knock out construct. This figure illustrates the PCR approach to determine that the knock out/-in construct has integrated into the DHFR-TS gene locus. Three different cells were tested: KI is a wildtype control, KII are cells transfected with a plasmid carrying only the disrupting cassettes but no DHFR-TS gene sequences and pKOI $D_{V L}$ are cells transformed with the $\mathrm{PKOI} \mathrm{D}_{\mathrm{VL}}$ plasmid. PCRI is a control reaction amplyfing 369 bp of the beta-hexosaminidase gene. PCR2 is to detect endogenous DHFR-TS (note that in the PKOID ${ }_{\mathrm{VL}}$ cells there still is a wildtype gene in the MIC). PCR3 only yields PCR-product for correctly integrated $\mathrm{PKOI} \mathrm{D}_{\mathrm{VL}}$ DNA. PCR4 shows that the full-length expression cassette has integrated. The first scheme illustrate the overall structure of the DHFR-TS gene, exons are coloured in green and introns in blue. The parts of the non-coding 5' and 3' regions of the DHFR-TS structure gene that we used as integration sites in the pKOI/ pKOI $D_{V L}$ plasmids are coloured in grey. The next scheme gives on overall structure of the pKOID $D_{\mathrm{VL}}$ expression construct that was used for transformation and integration into the MAC. The third scheme illustrates the disruption of the endogenous DHFR-TS structure gene by proper integration of the resistance and expression cassettes into the MAC. 


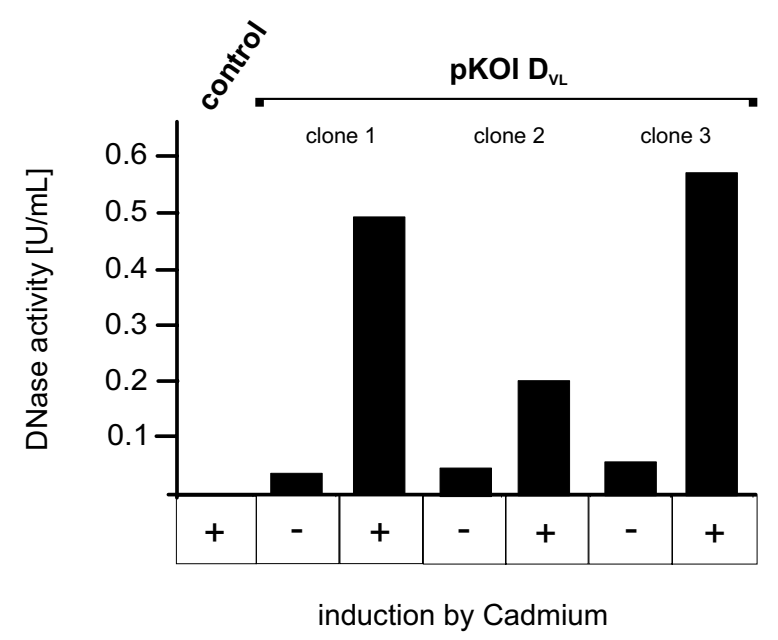

Figure 7

Enzyme function of knock in target. Supernatants of three clones transformed with $\mathrm{pKOI} \mathrm{D}_{\mathrm{VL}}$ were assayed for DNase activity. Only heavy metal induced cells (+) show high levels of DNase activity. Transformed, but non-induced cells show a slightly elevated enzyme activity compared to wildtype cells due to low basal promoter activity.

ing the organism's properties (for a short and comprehensive review see[26]).

It is well known that altering the cell's phenotype ultimately needs direct or indirect (by MIC transformation and conjugation) genetic engineering of the vegetative MAC. The first approaches for heterologous expression were done by using plasmids that use the vast amplification of the rDNA gene during anlagen/MAC development. However, the episomal presence of these plasmids depends on the drug concentration and the plasmid may recombine homologously and non-directionally into endogenous rDNA units.

Stable integration of expression cassettes into diploid MIC can be achieved by biolistic bombardment. After conjugation of two different mating types the old MACs disappear and new ones form that carry the new information derived from the recombinant MIC. The whole process follows the statistics of the Mendelian genetics. The advantage of this approach is that one obtains stable clones that maintain the genetic properties and that can be crossed via classical genetics to combine various properties of different $T$. thermophila strains. This approach is elaborative and time consuming. Furthermore, it has been shown recently that scan RNAs (snRNA) derived from the old MAC play an important role in DNA elimination during the development of the somatic MAC from the germline MIC (see [27] for review). The primary sequence of these small RNAs explains how the parental MAC epige-

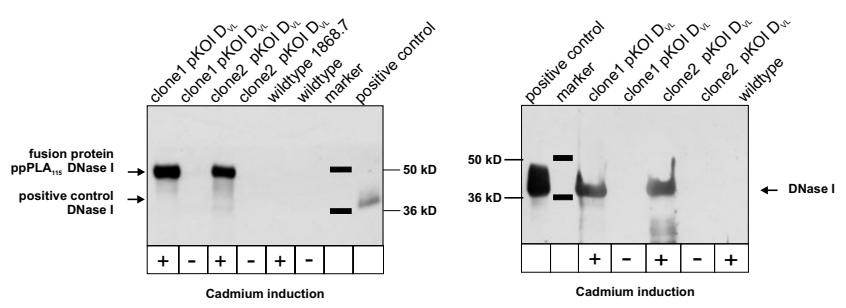

Figure 8

Expression of knock in target. Western blots show expression of recombinant human DNase I: Only transformed and heavy metal induced cells $(+)$ show strong signals due to anti-DNase I antibodies. The intracellular PLA I- $^{-}$ DNase-fusion protein is visualized on the left blot by samples of cell lysates. Bands are running at higher molecular weight than the mature and processed positive control due to the pre/pro-peptide. On the right, supernatants were subjected to Western blot; the size of the secreted protein of $T$. thermophila argues for a correct processing when compared to the positive control.

netically controls the genome rearrangement in the new MAC. In the case of stable MIC transformants this RNAilike mechanism may cause partial deletion of foreign expression cassettes in the developing new MAC [28].

Therefore instead of episomal transformation by rDNA based plasmids or the stable transformation of the MIC we used a shortcut by combining MAC transformation with allelic assortment. This combination has several advantages. Firstly, the MAC transformation is much more efficient because there are at least about 45 potential integration sites per gene locus. This increases the probability of integration to at least one order of magnitude. Secondly, not only conjugating but also non-conjugating and therefore defined strains can be transformed. This is very important, because it allows the stepwise improvement of strains by maintaining defined genetic properties. Thirdly after completed allelic assortment the use of any antibiotic is obsolete as wildtype alleles have vanished in a one-way-manner. This allows cheap cultivation at large scales for e.g. biotechnical production processes.

\section{Conclusion}

In summary use of the DHFR-TS gene locus as a target for homologous genomic integration does not only provide a robust and simple marker system to assure complete allelic assortment of altered ARPs in the MAC but also the pKOI concept combining the DHFR-TS knock out with an additional knock in and subsequent expression of foreign genes. 


\section{Methods \\ Cells and cell culture}

Tetrahymena thermophila strains B 1868/4, B 1868/7 and B $2068 / 1$ were cultivated in skimmed milk medium[29] in SPP ( $0.5 \%$ proteose peptone, $0.5 \%$ yeast extract, $0.1 \%$ ferrous sulphate chelate solution and $1 \%$ glucose) or in CDM medium[18].

\section{Amplification of the DHFR-TS gene of T. thermophila The DHFR-TS cDNA, gene including 5'and 3' flanking sites can be amplified using the following primer pairs. Nucleotides in small letters encode sites for restriction endonucleases. Amplification of the DHFR-TS 5' flanking region:}

DHFR 5'1 F NotI: 5'-cccgcggccgcACAGAGTTAATGGAAATGGAGC-3'

DHFR 5'2R BamHI: 5'-gggggatccATATTTAAGCGATCTTTCAATGG-3'

Amplification of the DHFR-TS CDNA and gene with introns:

\section{DHFR CDS-F:}

5'-cgcGAATTCATGAAAACAAGACATTTTGATATAGTTTTAGC-3'

\section{DHFR CDS-R:}

5'-gcgCTCGAGTCAGACAGCCATTTTCATTTATATTTTAGGG-3'

Amplification of the DHFR-TS 3 'flanking region:

DHFR 3'1F Xhol: $\quad$ 5'-gggctcgagATGCTCATGTT-
TACTCTAATCACG-3'

DHFR 3'1R Acc65I: 5'-gggggtaccAGTAAAAATAGAGTAGAAGGAG-3'.

\section{Construction of plasmids \\ Construction of $\mathrm{pKOI}$}

The pKOI (knock out/-in) plasmid was constructed as follows: As backbone for selection and propagation in E. coli. the pBlueScript II SK plasmid was used. The $1.5 \mathrm{~kb} 5{ }^{\prime}-$ DHFR-TS integration site was amplified using the primer pair DHFR 5' 1 F NotI and DHFR 5' 2 R BamHI cloned into pBS II SK by using NotI and BamHI sites. Next the 1.4 $\mathrm{kb}$ paromomycin selection cassette from the pH4T2 (neo2) was cloned into the intermediate pBS IISK by BamHI and SmaI sites. Finally, the 3'-DHFR-TS integration site was amplified by primers DHFR 3' $1 \mathrm{~F}$ XhoI and DHFR 3' 1 R Acc65I and cloned by using the Xhol and
Acc65I sites to finish the DHFR-TS knock out cassette. The SacI site of the pBS II SK backbone had been destroyed by site directed mutagenesis to facilitate the use of the endogenous SacI site in the 3'-DHFR-TS integrating sequence and the Xhol site as unique cloning site in pKOI. The whole $\mathrm{pKOI}$ basis vector is $\sim 7.7 \mathrm{~kb}$ in size and contains a multiple cloning site (figure 3 ).

\section{Construction of $\mathrm{PKOI} \mathrm{D}_{V L}$}

The unique XhoI and SacI sites were used to insert the ppPLA $_{115}$-DNase knock in expression cassette ( $\left.2.5 \mathrm{~kb}\right)$. This cassette encodes a fusion protein of the first 115 aa of the endogenous PLA 1 precursor and the aa 23-281 of the mature human DNaseI, flanked by a 1 kb MTT1 promotor active sequence and the $\sim 0.4 \mathrm{~kb}$ BTU2 terminator, leading to $\mathrm{a} \sim 10.2 \mathrm{~kb}$ vector. To ensure proper translation of this fusion protein a codon optimised synthetic human DNase I gene was used (submitted at BMC Biotechnol.)

The junction sequence between the MTT1 promoter and the precursor sequence of $\mathrm{PLA}_{1}$ is given with the sequence ATGgatatcAAC, using a EcoRV site (gatatc) between the initial ATG of the MTT1 gene and the second codon (AAC) of the $\mathrm{PLA}_{1}$ precursor sequence. The cDNA that encodes the ppPLA 115 -DNase I fusion protein ends with the TGA stop codon followed by a BglII site (agatct). Therefore the "knock in" cassette (generated in an intermediate vector) offers a modular structure that allows the simple replacement of the promoter (by Xhol/EcoRV), the coding sequence (by EcoRV/BglII) and the terminator DNA sequences (by BglII/SacI). Further details of the primers and constructs are available from the authors.

Transformation of pKOI plasmids (biolistic bombardment) We used conjugating cells, as well as vegetative, growing or stationary T. thermophila strains. The transformation of the $T$. thermophila cells was performed as previously described in[9].

Selection, allelic assortment and DHFR-TS knock out assay T. thermophila cell proliferation assay: For the first ca $16 \mathrm{~h}$ after biolistic bombardment transformants were grown in skimmed milk medium. After that transformed cells were grown on SPP medium with in increasing concentrations of paromomycin (from $100 \mu \mathrm{g} / \mathrm{mL}$ to $1000 \mu \mathrm{g} / \mathrm{mL}$ ) to support the allelic assortment process. After 3-4 weeks each clone was cultivated on CDM replica plates with or without thymidine $(10 \mathrm{mg} / \mathrm{mL})$. Functional DHFR-TS knock out clones are only able to grow in CDM medium supplemented with thymidine. The viability of the DHFRTS knock out strains was monitored by determining the growth kinetic. The complete integration of the DHFR-TS knock out and DNase I knock in cassette was confirmed by PCR using the following primers: 
DHFR01F: 5'-CTTTTTAACAGCCTGCTGCTCG-3'

DHFR02R: 5'-GATTTTGATGCTTCAATAAGGTTG-3'

DHFR03F: 5'-TTATTTGTTTTATCATAGTGGAAAAGG-3'

DHFR04R: 5'-CAGACACCTCAATCATATCAAAG-3'

DHFR05F: 5'-GGTCCTCCATCAGATTGTGG-3'

DHFR06R: 5'-CGCGTCGAGTCAGACAGCCATTT-

TCATTTA-3'

Hex01F: 5'-ATGCAAAAGATACTTTTAATTACTTTC-3'

Hex02R: 5'-TATATTTTAGGAATGTTGTAATC-3'

A pH4T2 plasmid carrying the same neo2 and DNase I expression/secretion cassettes was used as PCR control.

\section{SDS-PAGE and Western blot}

Aliquots of transformed cells and of SPP supernatants were resuspended in sample buffer and separated on $15 \%$ SDS-PAGE. The gels were blotted onto nitrocellulose membranes and blocked in PBS containing $0.05 \%$ Tween 20 and $5 \%$ skim milk (PBS-TM). The expression of recombinant human DNase I in transformed Ciliates was detected by two specific anti sera from rabbit against human DNase I (antigen: recombinant human DNase I, Pulmozyme, Roche). Both sera detected the recombinant DNase I antigen. The serum was used in a 1:500 dilution in PBS-TM. After washing with PBS/T and applying an HRP-conjugated anti rabbit serum. The blots were developed by using chemiluminescence.

\section{DNase I activity assay}

The methyl green based DNase activity assay was performed as already published [30]. Samples were incubated at $37^{\circ} \mathrm{C}$ for $24 \mathrm{~h}$ on a microtiter plate. Absorbance was measured at $620 \mathrm{~nm}$. Calibration of the assay was achieved by different amounts of defined DNase I Units of Pulmozyme from Roche (CHO derived) in each experiment and linear regression. These results combined with semi-quantitative Western blotting were used to calculate the specific activity of expressed DNase I.

\section{Sequence alignments}

DHFR-TS sequences were aligned with Sci Ed Central's Clonemanger Professional Suite v6.0 using the BLOSUM 62 score matrix.

\section{Authors' contributions}

LH evaluated the DHFR-TS targeting sequences, setup and cloned the pKOI vector system and participated in manuscript drafting. UB participated in performing the experi- ments (cloning of pKOI $\mathrm{D}_{\mathrm{VL}}$ expression constructs, transformation and screening of the ciliates, including monitoring of foreign gene expression) and in figure preparation. MWWH conceived of the study and participated in its design and coordination. TW participated in project conception, preparation of figures, did sequence alignments and was involved in manuscript drafting. All authors read and approved the final manuscript.

\section{Acknowledgements}

We would like to thank Angelika Kronenfeld for excellent technical assistance and commitment. This work represents main parts of the $\mathrm{PhD}$ thesis of UB.

\section{References}

I. Karrer KM: Tetrahymena genetics: two nuclei are better than one. Methods Cell Biol 2000, 62:127-186.

2. Gibbons IR, A.J R: Dynein: a protein with adenosine triphosphatase activity from cilia. Science 1965, I49:424-426.

3. Blackburn EH, Gall JG: A tandemly repeated sequence at the termini of the extrachromosomal ribosomal RNA genes in Tetrahymena. J Mol Biol 1978, I 20:33-53.

4. Cech TR, Zaug AJ, Grabowski PJ: In vitro splicing of the ribosomal RNA precursor of Tetrahymena: involvement of a guanosine nucleotide in the excision of the intervening sequence. Cell I98I, 27:487-496.

5. Greider $\mathrm{CW}$, Blackburn $\mathrm{EH}$ : Identification of a specific telomere terminal transferase activity in Tetrahymena extracts. Cell 1985, 43:405-4I3.

6. Brownell JE, Zhou J, Ranalli T, Kobayashi R, Edmondson DG, Roth SY, Allis CD: Tetrahymena histone acetyltransferase $A: a$ homolog to yeast Gcn5p linking histone acetylation to gene activation. Cell 1996, 84:843-85I.

7. Pan WC, Blackburn EH: Single extrachromosomal ribosomal RNA gene copies are synthesized during amplification of the rDNA in Tetrahymena. Cell I 98I, 23:459-466.

8. Gaertig J, Gorovsky MA: Efficient mass transformation of Tetrahymena thermophila by electroporation of conjugants. Proc Natl Acad Sci U S A 1992, 89:9196-9200.

9. Cassidy-Hanley D, Bowen J, Lee JH, Cole E, VerPlank LA, Gaertig J, Gorovsky MA, Bruns PJ: Germline and somatic transformation of mating Tetrahymena thermophila by particle bombardment. Genetics 1997, 146:135-147.

10. Larson DD, Blackburn EH, Yaeger PC, Orias E: Control of rDNA replication in Tetrahymena involves a cis-acting upstream repeat of a promoter element. Cell 1986, 47:229-240.

II. Hai B, Gaertig J, Gorovsky MA: Knockout heterokaryons enable facile mutagenic analysis of essential genes in Tetrahymena. Methods Cell Biol 2000, 62:513-531.

12. Gaertig J, Kapler G: Transient and stable DNA transformation of Tetrahymena thermophila by electroporation. Methods Cell Biol 2000, 62:485-500.

13. Clark TG, Gao Y, Gaertig J, Wang X, Cheng G: The I-antigens of Ichthyophthirius multifiliis are GPI-anchored proteins. J Eukaryot Microbiol 200I, 48:332-337.

14. Peterson DS, Gao Y, Asokan K, Gaertig J: The circumsporozoite protein of Plasmodium falciparum is expressed and localized to the cell surface in the free-living ciliate Tetrahymena thermophila. Mol Biochem Parasitol 2002, I 22: I 19-126.

15. Sweeney R, Fan Q, Yao MC: Antisense ribosomes: rRNA as a vehicle for antisense RNAs. Proc Natl Acad Sci U S A 1996, 93:8518-8523.

16. Guberman A, Hartmann M, Tiedtke A, Florin-Christensen J, FlorinChristensen M: A method for the preparation of Tetrahymena thermophila phospholipase AI suitable for large-scale production. J Appl Microbiol 1999, 86:226-230.

17. Wheatley DN, Rasmussen L, Tiedtke A: Tetrahymena: a model for growth, cell cycle and nutritional studies, with biotechnological potential. Bioessays 1994, I 6:367-372.

18. Hellenbroich D, Valley U, Ryll T, Wagner R, Tekkanat N, Kessler W, Ross A, Deckwer WD: Cultivation of Tetrahymena ther- 
mophila in a $1.5-\mathrm{m} 3$ airlift bioreactor. Appl Microbiol Biotechnol 1999, 5 I:447-455.

19. Gaertig J, Gu L, Hai B, Gorovsky MA: High frequency vectormediated transformation and gene replacement in Tetrahymena. Nucleic Acids Res 1994, 22:539I-5398.

20. Gaertig J, Thatcher TH, Gu L, Gorovsky MA: Electroporationmediated replacement of a positively and negatively selectable beta-tubulin gene in Tetrahymena thermophila. Proc Natl Acad Sci U S A 1994, 9 I:4549-4553.

21. Huennekens FM: The methotrexate story: a paradigm for development of cancer chemotherapeutic agents. Adv Enzyme Regul 1994, 34:397-419.:397-419.

22. Stechmann A, Cavalier-Smith T: Rooting the eukaryote tree by using a derived gene fusion. Science 2002, 297:89-9l.

23. The Institute for Genomic Research. [ http://www.tigr.org ]

24. Weide T, Herrmann L, Bockau U, Niebur N, Aldag I, Laroy W, Contreras A, Tiedtke A, Hartmann MWW: Secretion of functional human enzymes by Tetrahymena thermophila. BMC Biotechnol 2006, 6:19.

25. Shang $Y$, Song X, Bowen J, Corstanje R, Gao Y, Gaertig J, Gorovsky MA: A robust inducible-repressible promoter greatly facilitates gene knockouts, conditional expression, and overexpression of homologous and heterologous genes in Tetrahymena thermophila. Proc Natl Acad Sci U S A 2002, 99:3734-3739.

26. Collins K, Gorovsky MA: Tetrahymena thermophila. Curr Biol 2005, I 5:R3|7-R3 I8.

27. Yao MC, Chao JL: RNA-Guided DNA Deletion in Tetrahymena: An RNAi-Based Mechanism for Programmed Genome Rearrangements. Annu Rev Genet 2005, .:

28. Yao MC, Fuller $P, X i X$ : Programmed DNA deletion as an RNAguided system of genome defense. Science 2003, 300:158I-I584.

29. Kiy T, Tiedtke A: Continuous high-cell-density fermentation of the ciliated protozoon Tetrahymena in a perfused bioreactor. Appl Microbiol Biotechnol 1992, 38: I41-146.

30. Sinicropi D, Baker DL, Prince WS, Shiffer K, Shak S: Colorimetric determination of DNase I activity with a DNA-methyl green substrate. Anal Biochem 1994, 222:351-358.

Publish with Bio Med Central and every scientist can read your work free of charge

"BioMed Central will be the most significant development for disseminating the results of biomedical research in our lifetime. "

Sir Paul Nurse, Cancer Research UK

Your research papers will be:

- available free of charge to the entire biomedical community

- peer reviewed and published immediately upon acceptance

- cited in PubMed and archived on PubMed Central

- yours - you keep the copyright

Submit your manuscript here:

http://www.biomedcentral.com/info/publishing_adv.asp
BiolMedcentral 